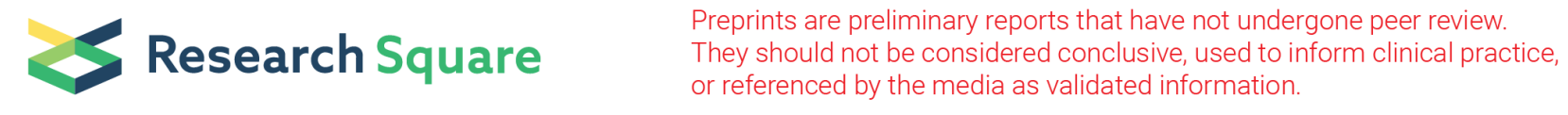

\title{
Value of visceral fat area in normal body mass index (BMI) patients is a predictive factor for peritoneal metastasis in gastric cancer: A prospective study
}

\author{
Chenchen Mao \\ Wenzhou Medical University Second Affiliated Hospital \\ Mingming Shi \\ Wenzhou Medical University First Affiliated Hospital \\ Hui Chen \\ Wenzhou Medical University Second Affiliated Hospital \\ Libin Xu \\ Wenzhou Medical University First Affiliated Hospital \\ He Huang \\ Wenzhou Medical University Second Affiliated Hospital
}

Xian Shen ( $\square$ shenxian5166@gmail.com )

The First Affiliated Hospital of Wenzhou Medical University https://orcid.org/0000-0003-1164-4832

\section{Research article}

Keywords: Gastric cancer; Peritoneal metastasis; Obesity; Visceral fat area; Body mass index

Posted Date: March 3rd, 2020

DOI: https://doi.org/10.21203/rs.3.rs-15896/v1

License: (c) (i) This work is licensed under a Creative Commons Attribution 4.0 International License. Read Full License 


\section{Abstract}

Background Although peritoneal metastasis (PM) is associated with poor prognosis in gastric cancer (GC) patients, it is difficult to discriminate preoperatively. Our previous study has demonstrated visceral fat area (VFA) is a better obesity index than body mass index (BMI) in predicting abdominal metastasis. Aim This study aimed to further explore the relationship between obesity and PM. Methods VFA was retrieved for 859 consecutive patients undergoing radical gastrectomy between January 1, 2009 and December 31, 2013. A receiver operating characteristic curve analysis was used to determine the BMI-specific cutoff values for VFA. Univariate and multivariate analyses evaluating the risk factors for PM at different BMI levels were performed. Results The optimal cutoff values for VFA were $67.28,88.03$, and $175.32 \mathrm{~cm} 2$ for low, normal, and high BMI patients, respectively, and 18 (15.52\%), 220 (40.15\%), and 61 (31.28\%) patients were classified as having high VFA in each group. Univariate logistic regression revealed that the association between high VFA and PM was not dependent on BMI (odds ratio [OR] $=9.048, P=0.007$ for low BMI, OR=3.827, $\mathrm{P}<0.001$ for normal $\mathrm{BMI}$, and $\mathrm{OR}=2.460, \mathrm{P}=0.049$ for high $\mathrm{BMI})$. In multivariate logistic regression analysis, high VFA $(\mathrm{OR}=3.816, \mathrm{P}<0.001)$ and vascular invasion ( $\mathrm{OR}=1.951, \mathrm{P}=0.039)$ were independent risk factors for $\mathrm{PM}$ only in the normal BMI group. Conclusions VFA only effectively predicted PM for GC patients with normal BMI, rather than those with low and high BMI. More attentions should be paid to those GC patients with high VFA and normal BMI.

\section{Introduction}

Gastric cancer (GC) is the fourth most common cancer worldwide and the third leading cause of cancer-related deaths (723000 deaths) worldwide ${ }^{[1,2]}$. Most GC patients do not succumb to their primary tumor but instead to peritoneal metastasis (PM), which is common in advanced gastric cancer ${ }^{[3]}$, but is even more apparent after the primary lesion has been removed ${ }^{[4]}$. Nearly $50 \%$ of the deaths due to GC could be ascribed to $\mathrm{PM}^{[5]}$ and patients with PM of GC experience poorer prognosis with a median survival of 4-7 months $s^{[6,7]}$. However, PM in GC may remain asymptomatic for a long period in most cases ${ }^{[6]}$ and is detected in only $14 \%$ of GC patients at initial examination ${ }^{[8]}$ and is often initially diagnosed intraoperatively, which is not ideal for surgeons to determine the most appropriate therapeutic approach. Thus, it is essential to develop effective methods to discriminate patients at a high risk of PM promptly.

Recently, obesity has also been recognized as an independent risk factor for several cancers ${ }^{[9]}$. It is reported that nearly $40 \%$ of all cancers can be attributed to overweight and obesity ${ }^{[10]}$. In particular, over $60 \%$ of endometrial, post-menopausal breast and colorectal cancers have been attributed to obesity ${ }^{[11,12]}$. However, obesity characterized by body mass index (BMI) is only a measure of general adiposity and may not fully quantify the role of visceral adiposity in cancer risk. Moreover, visceral adipose tissue is increasingly recognized as an endocrine organ that synthesizes obesity-mediated hormones and cytokines which have been directly implicated in cancer risk ${ }^{[13]}$. Furthermore, visceral fat area (VFA) has been demonstrated to be more strongly associated with postoperative complications such as incisional hernia ${ }^{[14]}$ and postsurgical gastroparesis syndrome ${ }^{[15]}$ after gastrointestinal operation than elevated BMI.

Similarly, our previous study also found that VFA was a more useful indicator of PM risk than BMI ${ }^{[16]}$. In this study, we evaluated the predictive value of the VFA for PM in gastric cancer patients with different BMI levels, and further explore the relationship between obesity and PM, via screening of patients at high risk of PM preoperatively.

\section{Materials And Methods Study design and patient population}

All patients who underwent radical gastrectomy at the Gastrointestinal Surgical Department, Second Affiliated Hospital of Wenzhou Medical University and the First Affiliated Hospital of Wenzhou Medical University in China between January 1, 2009 and December 31, 2013 were identified. Demographic information and operative details were prospectively collected and patients were grouped according to their BMI into "Iow BMI (BMI< $18.5 \mathrm{~kg} / \mathrm{m}^{2}$ )," "normal BMI (BMI 18.5-24 kg/m²)," and "high BMI (BMI $\left.\geq 24 \mathrm{~kg} / \mathrm{m}^{2}\right)$ " groups. All participants provided written informed consent prior to study participation. This project was approved by the Ethical Review Board of the Second Affiliated Hospital of Wenzhou Medical University.

\section{Inclusion/exclusion Criteria}

All adult patients who met the following criteria were included for analysis: (a) histopathologically confirmed gastric adenocarcinoma and planned to receive radical gastrectomy; (b) adult patients (18 years of age or older); (c) had abdominal computed tomography (CT) image within 1 month before surgery; (d) signed informed consent and agreed to participate in this study. The exclusion criteria included patients who: (a) lacked preoperative abdominal computed tomography (CT) image (performed CT in other hospital); (b) lacked the data on weight or height needed to calculate BMI; (c) had cancer metastasis which could not be cured during surgery; (d) patients who had undergone partial gastrectomy with remnant GC. Operation were performed routinely following the Japanese Gastric Cancer Treatment Guidelines 2010 (version 3).

\section{Diagnosis Of Pm}


PM was diagnosed according to the criteria of the Japanese Gastric Cancer Treatment Guidelines (15th edition): metastases were limited to the greater omentum, lesser omentum, anterior lobe of the transverse mesocolon, pancreatic capsule, and spleen and metastasis in the upper abdominal peritoneum (visceral peritoneum above the transverse position and parietal peritoneum above the umbilicus). PM was diagnosed from intraoperative frozen sections and postoperative diagnostic pathology.

\section{Vfa Measurement}

As mentioned above, all patients received abdominal CT scan preoperatively. The umbilicus level imaging was selected for measurement from the Picture Archiving and Communication System (PACS). The Hounsfield scale was used to distinguish adipose tissue from other tissues; here, adipose tissue was defined as within the range of -140 to -50 Hounsfield units (HU). The total fat area was calculated using a dedicated processing system (version 3.0.11.3, BN17 32-bit; INFINITT Healthcare Co., Ltd., Seoul, South Korea).

\section{Cutoff Point For Vfa}

We determined the cutoff point for VFA as the maximal Youden index value on a receiver operating characteristic (ROC) curve. Patients in each subgroup were then divided into two groups based on this cutoff point-the "high VFA group" and the "low VFA group."

\section{Statistical analysis}

The Kolmogorov-Smirnov test was performed to assess the distribution equality of continuous parameters. Normally distributed data are presented as mean \pm standard deviation (SD), whereas non-normally distributed data are presented as medians and interquartile ranges (IQRs). Continuous normally distributed data were compared using t-test while Mann-Whitney U test was used for continuous, non-normally distributed data. Categorical data were compared using the chi-squared test or Fisher's exact test. Univariate logistic regression analysis of all potential baseline predictors was performed to compute the odds ratios (ORs) with their $95 \%$ confidence intervals $(\mathrm{Cls})$. Variables with a trend $(\mathrm{P}<0.05)$ in the univariate analysis were selected as potential parameters, and then, a forward stepwise variable selection was used to establish a multivariable logistic regression model. All Pvalues were two-sided, and $\mathrm{P}<0.05$ was considered statistically significant. All statistical analyses were performed using SPSS software (version 22.0 ; SPSS Inc., Chicago, IL, USA) and R software (version 3.0.1; http://www.Rproject.org).

\section{Results}

An overview of the patient population is provided in Table 1. A total of 859 patients met the inclusion criteria and were enrolled in the analysis and 87 (10.13\%) patients were diagnosed with PM as per the criteria mentioned above. The mean age of the patients was $63.63 \pm 11.28$ years and majority of them were men $(672,78.2 \%)$. The mean VFA and tumor size of the patients were $89.60 \pm 60.91 \mathrm{~cm}^{2}$ and $3.88 \pm 2.23 \mathrm{~cm}$, respectively. Other Clinical and demographic characteristics of the cohort have been reported in our previous study. 


\begin{tabular}{|c|c|c|c|c|c|}
\hline \multirow[t]{2}{*}{ Characteristic } & \multirow[t]{2}{*}{ Overall } & \multicolumn{3}{|c|}{ Level of BMI $\left(\mathrm{kg} / \mathrm{m}^{2}\right)$} & \multirow[t]{2}{*}{$\mathrm{P}$} \\
\hline & & Low BMI ( $₫ 18.5)$ & Normal BMI (18.5-24) & High BMI $(\geq 24)$ & \\
\hline No. patients & 859 & 116 & 548 & 195 & \\
\hline Age (y) & $63.63 \pm 11.28$ & $67.10 \pm 10.97$ & $63.55 \pm 11.25$ & $61.77 \pm 11.14$ & $<0.001 *$ \\
\hline VFA $\left(\mathrm{cm}^{2}\right)$ & $89.60 \pm 60.91$ & $38.67 \pm 31.94$ & $79.43 \pm 49.74$ & $148.45 \pm 59.20$ & $<0.001 *$ \\
\hline Gender $[n,(\%)]$ & & & & & 0.273 \\
\hline Male & $672(78.23 \%)$ & $88(75.86 \%)$ & 438 (79.93\%) & $146(74.87 \%)$ & \\
\hline Female & $187(21.77 \%)$ & $28(24.14 \%)$ & $110(20.07 \%)$ & $49(25.13 \%)$ & \\
\hline ASA score $[n,(\%)]$ & & & & & 0.162 \\
\hline$\square 3$ & 759 (88.36\%) & 97 (83.62\%) & 485 (88.50\%) & 177 (90.77\%) & \\
\hline$\geq 3$ & $100(11.64 \%)$ & $19(16.38 \%)$ & $63(11.50 \%)$ & $18(9.23 \%)$ & \\
\hline Charlson score $[\mathrm{n},(\%)]$ & & & & & $0.010^{*}$ \\
\hline 0 & $504(58.67 \%)$ & $72(62.07 \%)$ & $331(60.40 \%)$ & $101(51.79 \%)$ & \\
\hline $1-3$ & $333(38.77 \%)$ & $39(33.62 \%)$ & $210(38.32 \%)$ & $84(43.08 \%)$ & \\
\hline $4-6$ & $22(2.56 \%)$ & $5(4.31 \%)$ & $7(1.28 \%)$ & $10(5.13 \%)$ & \\
\hline Preoperative hypoproteinemia [n, (\%)] & & & & & $<0.001 *$ \\
\hline No & 725 (84.40\%) & $84(72.41 \%)$ & $466(85.04 \%)$ & $175(89.74 \%)$ & \\
\hline Yes & $134(15.60 \%)$ & $32(27.59 \%)$ & $82(14.96 \%)$ & $20(10.26 \%)$ & \\
\hline Preoperative anemia $[\mathrm{n},(\%)]$ & & & & & $<0.001 *$ \\
\hline No & $424(49.36 \%)$ & $40(34.48 \%)$ & 255 (46.53\%) & 129 (66.15\%) & \\
\hline Yes & 435 (50.64\%) & $76(65.52 \%)$ & 293 (53.47\%) & 66 (33.85\%) & \\
\hline Tumor size $(\mathrm{cm})$ & $3.88 \pm 2.23$ & $4.29 \pm 2.38$ & $3.91 \pm 2.25$ & $3.53 \pm 2.04$ & $0.012 *$ \\
\hline Tumor location [n, (\%)] & & & & & 0.585 \\
\hline Cardia & $150(17.46 \%)$ & $16(13.79 \%)$ & $98(17.88 \%)$ & $36(18.46 \%)$ & \\
\hline Antrum & 579 (67.41\%) & $82(70.69 \%)$ & 362 (66.06\%) & $135(69.23 \%)$ & \\
\hline Other & $130(15.13 \%)$ & $18(15.52 \%)$ & $88(16.06 \%)$ & $24(12.31 \%)$ & \\
\hline Histopathological differentiation $[\mathrm{n},(\%)]$ & & & & & 0.616 \\
\hline Differentiated & $671(78.11 \%)$ & $87(75.00 \%)$ & $433(79.01 \%)$ & $151(77.44 \%)$ & \\
\hline Undifferentiated & $188(21.89 \%)$ & $29(25.00 \%)$ & $115(20.99 \%)$ & $44(22.56 \%)$ & \\
\hline Vascular invasion $[\mathrm{n},(\%)]$ & & & & & 0.166 \\
\hline No & $522(60.77 \%)$ & $63(54.31 \%)$ & 332 (60.58\%) & $127(65.13 \%)$ & \\
\hline Yes & $337(39.23 \%)$ & $53(45.69 \%)$ & $216(39.42 \%)$ & $68(34.87 \%)$ & \\
\hline Lymphatic invasion $[\mathrm{n},(\%)]$ & & & & & 0.06 \\
\hline No & $351(40.86 \%)$ & 45 (38.79\%) & 212 (38.69\%) & $94(48.21 \%)$ & \\
\hline Yes & $508(59.14 \%)$ & $71(61.21 \%)$ & $336(61.31 \%)$ & $101(51.79 \%)$ & \\
\hline Invasion depth $[\mathrm{n},(\%)]$ & & & & & $0.004^{*}$ \\
\hline T1 & 196 (22.82\%) & $17(14.66 \%)$ & $118(21.53 \%)$ & $61(31.28 \%)$ & \\
\hline $\mathrm{T} 2$ & $93(10.83 \%)$ & $12(10.34 \%)$ & $58(10.58 \%)$ & $23(11.79 \%)$ & \\
\hline T3 & $218(25.38 \%)$ & $42(36.21 \%)$ & $135(24.64 \%)$ & $41(21.03 \%)$ & \\
\hline $\mathrm{T} 4$ & $352(40.98 \%)$ & 45 (38.79\%) & $237(43.25 \%)$ & $70(35.90 \%)$ & \\
\hline Ulcer type & & & & & 0.518 \\
\hline No & 300 (34.92\%) & 36 (31.03\%) & $191(34.85 \%)$ & 73 (37.44\%) & \\
\hline Yes & 559 (65.08\%) & 80 (68.97\%) & 357 (65.15\%) & $122(62.56 \%)$ & \\
\hline Abdominal surgery history $[\mathrm{n},(\%)]$ & & & & & 0.144 \\
\hline No & 768 (89.41\%) & 99 (85.34\%) & 498 (90.88\%) & 171 (87.69\%) & \\
\hline Yes & $91(10.59 \%)$ & $17(14.66 \%)$ & $50(9.12 \%)$ & $24(12.31 \%)$ & \\
\hline Peritoneal metastasis $[\mathrm{n},(\%)]$ & & & & & $<0.001 *$ \\
\hline No & 772 (89.87\%) & 109 (93.97\%) & 490 (89.42\%) & $134(68.72 \%)$ & \\
\hline Yes & $87(10.13 \%)$ & $7(6.02 \%)$ & $58(10.58 \%)$ & $61(31.28 \%)$ & \\
\hline
\end{tabular}

Table.1 Characteristics of the Patients

Data presented as mean \pm SD and as \% demographic parameters in the entire study population by level of BMI.

BMI indicates body mass index; VFA, visceral fat area; ASA, American Society of Anesthesiologists.

*Statistically significant.

\section{Clinical And Demographic Characteristics In Different Bmi Group}

Patients were subdivided into low BMI group (BMI $\left.<18.5 \mathrm{~kg} / \mathrm{m}^{2}\right)$, normal BMI group (BMI 18.5-24 kg/m²) and high BMI group (BMI $\left.\geq 24 \mathrm{~kg} / \mathrm{m}^{2}\right)$, and each group contained 116, 548, and 195 patients, respectively. As shown in Table 1, patients in the low BMI group were older (P<0.001) and more likely to have lower VFA $(P<0.001)$ and larger tumor size $(P=0.012)$ compared to those in the normal and high $B M I$ groups. Univariate associations were found between BMI level and some clinical and demographic characteristics. The rates for preoperative hypoproteinemia $(\mathrm{P}<0.001)$ and preoperative anemia $(P<0.001)$ increased with an increasing level of BMI. Higher Charlson score $(P=0.010)$ and deeper invasion depth $(P=0.004)$ were found with increasing BMI. The frequency of PM was significantly higher among patients with high BMI compared to those with low and high (31.28\% vs $6.02 \%$ vs $10.58 \%$, respectively, $\mathrm{P}<0.001)$.

\section{Vfa Characteristics}

The overview of the VFA level in different BMI group is shown in Fig. 1. Patients with PM of GC had a significantly higher VFA in the normal BMI group $(P<0.001)$ and in high BMI group $(P=0.030)$ compared to those without $P M$. Although no statistical significance was found, patients with $P M$ were more likely to have higher VFA than those without in the low BMI group.

\section{Cutoff Values For Vfa In Different Bmi Groups}

According to the study including criteria, 116 of low BMI patients, 548 of normal BMI patients and 195 of high BMI patients were subdivided (Table 1). ROC curves of VFA were used to identify PM in each of the three groups, and the AUC were $0.658,0.616$, and 0.685 (Fig. 2), respectively. The optimal cutoff values for VFA were $67.28,88.03$, and $175.32 \mathrm{~cm}^{2}$ for patients with low, normal and high BMI, respectively. Using these cutoff values, 18 (15.52\%), 220 (40.15\%), and 61 (31.28\%) patients were classified as high VFA in each group. 
As shown in Table 2, PM was associated with high VFA $(P=0.007)$, histopathological differentiation $(P=0.012)$, and abdominal surgery history $(P=$ $0.046)$ in the low BMI cohort in univariate analysis. However, in multivariate logistic regression analysis, none of the three characteristics achieved statistical significance. In contrast, the univariate analysis identified VFA $(P<0.001)$, tumor size $(P=0.016)$, vascular invasion $(P<0.001)$, lymphatic invasion $(P=0.001)$, and invasion depth $(P=0.376$ for $T 2, P=0.011$ for $T 3$, and $P=0.002$ for $T 4)$ as factors that correlated significantly with $P M$ in normal BMI patients. Subsequent multivariate logistic regression analysis identified VFA $(O R=3.816, P<0.001)$ and vascular invasion $(O R=1.951, P=$ $0.039)$ as independent risk factors for $P M$. For the high BMI group, VFA $(P=0.049)$, Charlson score $(P=0.030)$, tumor size $(P=0.001)$, vascular invasion $(P<0.001)$, lymphatic invasion $(P=0.043)$, invasion depth $(P=0.163$ for $T 2, P=0.021$ for $T 3$, and $P=0.017$ for $T 4)$, and abdominal surgery history $(P=0.030)$ were statistically significant in the univariate analysis, and only vascular invasion $(O R=4.460, P=0.028)$ and abdominal surgery history $(\mathrm{OR}=5.321, \mathrm{P}=0.023)$ were independent predictors of $\mathrm{PM}$ in multivariate logistic regression analysis. 
Table 2

Univariate and Multivariate Analysis to Evaluate Potential Predictive Factors for Peritoneal Metastasis in patients with different BMI.

\begin{tabular}{|c|c|c|c|c|c|c|c|c|c|c|c|c|}
\hline \multirow[t]{5}{*}{ Factors } & \multicolumn{4}{|c|}{ Low BMI $\left(\bigotimes 18.5 \mathrm{~kg} / \mathrm{cm}^{2}\right)$} & \multicolumn{4}{|c|}{ Normal BMI $\left(18.5-24 \mathrm{~kg} / \mathrm{cm}^{2}\right)$} & \multicolumn{4}{|c|}{ High BMI ( $\left.\geq 24 \mathrm{~kg} / \mathrm{cm}^{2}\right)$} \\
\hline & \multicolumn{2}{|c|}{$\begin{array}{l}\text { Univariate } \\
\text { analysis }\end{array}$} & \multicolumn{2}{|c|}{$\begin{array}{l}\text { Multivariate } \\
\text { analysis }\end{array}$} & \multicolumn{2}{|c|}{$\begin{array}{l}\text { Univariate } \\
\text { analysis }\end{array}$} & \multicolumn{2}{|c|}{$\begin{array}{l}\text { Multivariate } \\
\text { analysis }\end{array}$} & \multicolumn{2}{|c|}{ Univariate analysis } & \multicolumn{2}{|c|}{$\begin{array}{l}\text { Multivariate } \\
\text { analysis }\end{array}$} \\
\hline & OR & $\mathbf{P}$ & OR & $\mathbf{P}$ & OR & $\mathbf{P}$ & OR & $\mathbf{P}$ & OR $(95 \%$ & $\mathbf{P}$ & OR & $\mathbf{P}$ \\
\hline & $(95 \%$ & & $(95 \%$ & & $(95 \%$ & & $(95 \%$ & & $\mathrm{Cl})$ & & $(95 \%$ & \\
\hline & $\mathrm{Cl})$ & & $\mathrm{Cl})$ & & $\mathrm{Cl})$ & & $\mathrm{Cl})$ & & & & $\mathrm{Cl})$ & \\
\hline \multicolumn{13}{|l|}{ Age } \\
\hline$\triangle 65$ & 1 & & & & 1 & & & & 1 & & & \\
\hline$\geq 65$ & $\begin{array}{l}1.692 \\
(0.314- \\
9.115)\end{array}$ & 0.540 & & & $\begin{array}{l}0.993 \\
(0.575- \\
1.713)\end{array}$ & 0.979 & & & $\begin{array}{l}2.095 \\
(0.857- \\
5.125)\end{array}$ & 0.105 & & \\
\hline \multicolumn{13}{|l|}{ Gender } \\
\hline Female & 1 & & & & 1 & & & & 1 & & & \\
\hline Male & $\begin{array}{l}1.976 \\
(0.228- \\
17.152)\end{array}$ & 0.537 & & & $\begin{array}{l}2.335 \\
(0.976- \\
5.587)\end{array}$ & 0.057 & & & $\begin{array}{l}1.582 \\
(0.508- \\
4.924)\end{array}$ & 0.428 & & \\
\hline \multicolumn{13}{|l|}{ VFA } \\
\hline Low & 1 & & 1 & & 1 & & 1 & & 1 & & 1 & \\
\hline High & $\begin{array}{l}9.048 \\
(1.829- \\
44.753)\end{array}$ & $0.007 *$ & $\begin{array}{l}5.694 \\
(0.993- \\
32.654)\end{array}$ & 0.051 & $\begin{array}{l}3.827 \\
(2.130- \\
6.875)\end{array}$ & <. $0.001 *$ & $\begin{array}{l}3.816 \\
(2.091- \\
6.962)\end{array}$ & < $0.001 *$ & $\begin{array}{l}2.460 \\
(1.002- \\
6.039)\end{array}$ & $0.049 *$ & $\begin{array}{l}1.883 \\
(0.631- \\
5.624))\end{array}$ & 0.257 \\
\hline \multicolumn{13}{|c|}{ ASA score } \\
\hline$凶 3$ & 1 & & & & 1 & & & & 1 & & & \\
\hline$\geq 3$ & $\begin{array}{l}0.843 \\
(0.096- \\
7.428)\end{array}$ & 0.877 & & & $\begin{array}{l}1.265 \\
(0.570- \\
2.809)\end{array}$ & 0.563 & & & $\begin{array}{l}0.981 \\
(0.210- \\
4.586)\end{array}$ & 0.981 & & \\
\hline \multicolumn{13}{|c|}{ Charlson score } \\
\hline 0 & 1 & & & & 1 & & & & 1 & & 1 & \\
\hline $1-3$ & $\begin{array}{l}1.917 \\
(0.368- \\
9.983)\end{array}$ & 0.440 & & & $\begin{array}{l}1.544 \\
(0.893- \\
2.667)\end{array}$ & 0.120 & & & $\begin{array}{l}2.899 \\
(1.051- \\
8.000)\end{array}$ & $0.040 *$ & $\begin{array}{l}2.547 \\
(0.785- \\
8.265)\end{array}$ & 0.112 \\
\hline $4-6$ & $\begin{array}{l}5.750 \\
(0.483- \\
68.491)\end{array}$ & 0.166 & & & - & 0.999 & & & $\begin{array}{l}6.786 \\
(1.392- \\
33.086)\end{array}$ & $0.018^{*}$ & $\begin{array}{l}2.575 \\
(0.295- \\
22.512)\end{array}$ & 0.393 \\
\hline \multicolumn{13}{|c|}{$\begin{array}{l}\text { Preoperative } \\
\text { hypoproteinemia }\end{array}$} \\
\hline No & 1 & & & & 1 & & & & 1 & & & \\
\hline Yes & - & 0.998 & & & $\begin{array}{l}1.381 \\
(0.684- \\
3.095)\end{array}$ & 0.368 & & & $\begin{array}{l}1.449 \\
(0.388- \\
5.405)\end{array}$ & 0.581 & & \\
\hline \multicolumn{13}{|c|}{$\begin{array}{l}\text { Preoperative } \\
\text { anemia }\end{array}$} \\
\hline No & 1 & & & & 1 & & & & 1 & & & \\
\hline
\end{tabular}

Notes: * Statistically significant $(P<0.05)$.

Figure 1. Distribution of VFA between PM and Non-PM in different BMI groups.

Figure 2. ROC curves to identify PM in different BMI groups. The area under the ROC curve (AUC) values for VFA were shown.

Figure.1: 


\begin{tabular}{|c|c|c|c|c|c|c|c|c|c|c|c|c|}
\hline \multirow[t]{5}{*}{ Factors } & \multicolumn{4}{|c|}{ Low BMI ( $\left(18.5 \mathrm{~kg} / \mathrm{cm}^{2}\right)$} & \multicolumn{4}{|c|}{ Normal BMI (18.5-24 kg/cm²) } & \multicolumn{4}{|c|}{ High BMI $\left(\geq 24 \mathrm{~kg} / \mathrm{cm}^{2}\right)$} \\
\hline & \multicolumn{2}{|c|}{$\begin{array}{l}\text { Univariate } \\
\text { analysis }\end{array}$} & \multicolumn{2}{|c|}{$\begin{array}{l}\text { Multivariate } \\
\text { analysis }\end{array}$} & \multicolumn{2}{|c|}{$\begin{array}{l}\text { Univariate } \\
\text { analysis }\end{array}$} & \multicolumn{2}{|c|}{$\begin{array}{l}\text { Multivariate } \\
\text { analysis }\end{array}$} & \multicolumn{2}{|c|}{ Univariate analysis } & \multicolumn{2}{|c|}{$\begin{array}{l}\text { Multivariate } \\
\text { analysis }\end{array}$} \\
\hline & OR & $P$ & OR & $\mathbf{P}$ & OR & $\mathbf{P}$ & OR & $\mathbf{P}$ & OR (95\% & $\mathbf{P}$ & OR & $\mathbf{P}$ \\
\hline & $(95 \%$ & & $(95 \%$ & & $(95 \%$ & & $(95 \%$ & & $\mathrm{Cl})$ & & $(95 \%$ & \\
\hline & $\mathrm{Cl})$ & & Cl) & & $\mathrm{Cl})$ & & $\mathrm{Cl})$ & & & & $\mathrm{Cl})$ & \\
\hline Yes & $\begin{array}{l}0.685 \\
(0.146- \\
3.224)\end{array}$ & 0.632 & & & $\begin{array}{l}1.751 \\
(0.991- \\
3.095)\end{array}$ & 0.054 & & & $\begin{array}{l}1.741 \\
(0.710- \\
4.272)\end{array}$ & 0.226 & & \\
\hline \multicolumn{13}{|l|}{ Tumor size } \\
\hline$\varangle 3.5$ & 1 & & & & 1 & & 1 & & 1 & & 1 & \\
\hline$\geq 3.5$ & $\begin{array}{l}0.938 \\
(0.200- \\
4.394)\end{array}$ & 0.935 & & & $\begin{array}{l}2.048 \\
(1.142- \\
3.672)\end{array}$ & $0.016^{\star}$ & $\begin{array}{l}1.155 \\
(0.592- \\
2.250)\end{array}$ & 0.673 & $\begin{array}{l}8.473 \\
(2.417- \\
29.702)\end{array}$ & $0.001 *$ & $\begin{array}{l}3.955 \\
(0.842- \\
18.580)\end{array}$ & 0.082 \\
\hline \multicolumn{13}{|l|}{$\begin{array}{l}\text { Histopathological } \\
\text { differentiation }\end{array}$} \\
\hline Differentiated & 1 & & 1 & & 1 & & & & 1 & & & \\
\hline Undifferentiated & $\begin{array}{l}8.854 \\
(1.615- \\
48.528)\end{array}$ & $0.012^{\star}$ & $\begin{array}{l}5.425 \\
(0.809- \\
36.384)\end{array}$ & 0.082 & $\begin{array}{l}1.151 \\
(0.577- \\
2.298)\end{array}$ & 0.690 & & & $\begin{array}{l}1.011 \\
(0.350- \\
2.914)\end{array}$ & 0.984 & & \\
\hline \multicolumn{13}{|l|}{$\begin{array}{l}\text { Vascular } \\
\text { invasion }\end{array}$} \\
\hline No & 1 & & & & 1 & & 1 & & 1 & & 1 & \\
\hline Yes & $\begin{array}{l}1.633 \\
(0.349- \\
7.645)\end{array}$ & 0.534 & & & $\begin{array}{l}3.330 \\
(1.880- \\
3.095)\end{array}$ & $<.001 *$ & $\begin{array}{l}1.951 \\
(1.035- \\
3.680)\end{array}$ & $0.039 *$ & $\begin{array}{l}8.133 \\
(2.848- \\
23.226)\end{array}$ & $<.001 *$ & $\begin{array}{l}4.460 \\
(1.174- \\
16.942)\end{array}$ & $0.028^{*}$ \\
\hline \multicolumn{13}{|l|}{$\begin{array}{l}\text { Lymphatic } \\
\text { invasion }\end{array}$} \\
\hline No & 1 & & & & 1 & & 1 & & 1 & & 1 & \\
\hline Yes & $\begin{array}{l}0.232 \\
(0.043- \\
1.251)\end{array}$ & 0.089 & & & $\begin{array}{l}3.367 \\
(1.664- \\
6.811)\end{array}$ & $0.001^{\star}$ & $\begin{array}{l}1.773 \\
(0.786- \\
3.997)\end{array}$ & 0.168 & $\begin{array}{l}2.761 \\
(1.032- \\
29.702)\end{array}$ & $0.043^{\star}$ & $\begin{array}{l}1.321 \\
(0.363- \\
4.810)\end{array}$ & 0.673 \\
\hline \multicolumn{13}{|l|}{ Invasion depth } \\
\hline T1 & 1 & & & & 1 & & 1 & & 1 & & 1 & \\
\hline T2 & - & 1.000 & & & $\begin{array}{l}2.091 \\
(0.409- \\
10.696)\end{array}$ & 0.376 & $\begin{array}{l}1.268 \\
(0.235- \\
6.844)\end{array}$ & 0.782 & $\begin{array}{l}5.714 \\
(0.492- \\
66.309)\end{array}$ & 0.163 & $\begin{array}{l}1.774 \\
(0.105- \\
29.962)\end{array}$ & 0.691 \\
\hline T3 & - & 0.998 & & & $\begin{array}{l}5.154 \\
(1.463- \\
18.161)\end{array}$ & 0.011 & $\begin{array}{l}2.616 \\
(0.638- \\
10.730)\end{array}$ & 0.182 & $\begin{array}{l}12.353 \\
(1.458- \\
104.690)\end{array}$ & 0.021 & $\begin{array}{l}3.502 \\
(0.282- \\
43.514)\end{array}$ & 0.330 \\
\hline $\mathrm{T} 4$ & - & 0.998 & & & $\begin{array}{l}6.866 \\
(2.068- \\
22.792)\end{array}$ & 0.002 & $\begin{array}{l}2.839 \\
(0.703- \\
11.470)\end{array}$ & 0.143 & $\begin{array}{l}12.414 \\
(1.564- \\
98.542)\end{array}$ & 0.017 & $\begin{array}{l}1.511 \\
(0.106- \\
21.601)\end{array}$ & 0.761 \\
\hline \multicolumn{13}{|l|}{ Ulcer type } \\
\hline No & 1 & & & & 1 & & & & 1 & & & \\
\hline
\end{tabular}

Notes: * Statistically significant $(P<0.05)$.

Figure 1. Distribution of VFA between PM and Non-PM in different BMI groups.

Figure 2. ROC curves to identify PM in different BMI groups. The area under the ROC curve (AUC) values for VFA were shown.

Figure.1: 


\begin{tabular}{|c|c|c|c|c|c|c|c|c|c|c|c|c|}
\hline \multirow[t]{3}{*}{ Factors } & \multicolumn{4}{|c|}{ Low BMI ( $\left(18.5 \mathrm{~kg} / \mathrm{cm}^{2}\right)$} & \multicolumn{4}{|c|}{ Normal BMI $\left(18.5-24 \mathrm{~kg} / \mathrm{cm}^{2}\right)$} & \multicolumn{4}{|c|}{ High BMI ( $\left.\geq 24 \mathrm{~kg} / \mathrm{cm}^{2}\right)$} \\
\hline & \multicolumn{2}{|c|}{$\begin{array}{l}\text { Univariate } \\
\text { analysis }\end{array}$} & \multicolumn{2}{|c|}{$\begin{array}{l}\text { Multivariate } \\
\text { analysis }\end{array}$} & \multicolumn{2}{|c|}{$\begin{array}{l}\text { Univariate } \\
\text { analysis }\end{array}$} & \multicolumn{2}{|c|}{$\begin{array}{l}\text { Multivariate } \\
\text { analysis }\end{array}$} & \multicolumn{2}{|c|}{ Univariate analysis } & \multicolumn{2}{|c|}{$\begin{array}{l}\text { Multivariate } \\
\text { analysis }\end{array}$} \\
\hline & $\begin{array}{l}\text { OR } \\
(95 \% \\
\text { Cl) }\end{array}$ & $\mathbf{P}$ & $\begin{array}{l}\text { OR } \\
(95 \% \\
\mathrm{Cl})\end{array}$ & $\mathbf{P}$ & $\begin{array}{l}\text { OR } \\
(95 \% \\
\mathrm{Cl})\end{array}$ & $\mathbf{P}$ & $\begin{array}{l}\text { OR } \\
(95 \% \\
\mathrm{Cl})\end{array}$ & $\mathbf{P}$ & $\begin{array}{l}\text { OR }(95 \% \\
\mathrm{Cl})\end{array}$ & $\mathbf{P}$ & $\begin{array}{l}\text { OR } \\
(95 \% \\
\text { Cl) }\end{array}$ & $\mathbf{P}$ \\
\hline Yes & $\begin{array}{l}2.838 \\
(1.615- \\
48.528)\end{array}$ & 0.343 & & & $\begin{array}{l}1.055 \\
(0.257- \\
4.336)\end{array}$ & 0.941 & & & $\begin{array}{l}1.053 \\
(1.419- \\
2.647)\end{array}$ & 0.912 & & \\
\hline \multicolumn{13}{|c|}{$\begin{array}{l}\text { Abdominal } \\
\text { surgery history }\end{array}$} \\
\hline No & 1 & & 1 & & 1 & & & & 1 & & 1 & \\
\hline Yes & $\begin{array}{l}5.089 \\
(1.029- \\
25.173)\end{array}$ & 0.046 & $\begin{array}{l}1.823 \\
(0.259- \\
12.825)\end{array}$ & 0.546 & $\begin{array}{l}0.715 \\
(0.248- \\
2.064)\end{array}$ & 0.535 & & & $\begin{array}{l}3.229 \\
(1.121- \\
9.298)\end{array}$ & 0.030 & $\begin{array}{l}5.321 \\
(1.258- \\
22.510)\end{array}$ & $0.023^{*}$ \\
\hline \multicolumn{13}{|c|}{ Notes: * Statistically significant $(P<0.05)$. } \\
\hline \multicolumn{13}{|c|}{ Figure 1. Distribution of VFA between PM and Non-PM in different BMI groups. } \\
\hline \multicolumn{13}{|c|}{ Figure 2. ROC curves to identify PM in different BMI groups. The area under the ROC curve (AUC) values for VFA were shown. } \\
\hline Figure.1: & & & & & & & & & & & & \\
\hline
\end{tabular}

\section{Discussion}

Overweight and obesity are important lifestyle-related public health problems in the world ${ }^{[17]}$, and the number of overweight or obese people has already surpassed the number of underweight people for the first time according to recent studies ${ }^{[18]}$. In the literature, overweight and obesity is usually determined using the easily calculated BMI. BMI is classified as normal $\left(B M l<25 \mathrm{~kg} / \mathrm{m}^{2}\right.$ ), overweight (BMI $25-30 \mathrm{~kg} / \mathrm{m}^{2}$ ), and obese (BMI $\geq$ $\left.30 \mathrm{~kg} / \mathrm{m}^{2}\right)$ according to the WHO standard ${ }^{[19]}$. In this study, the Chinese standard ${ }^{[20,21]}$ was used and BMI was classified as low $\left(\mathrm{BMI}<18.5 \mathrm{~kg} / \mathrm{m}^{2}\right)$, normal (BMI $\left.18.5-24 \mathrm{~kg} / \mathrm{m}^{2}\right)$, high (BMI $\geq 24 \mathrm{~kg} / \mathrm{m}^{2}$ ), as all the patients enrolled were Chinese.

Recently, increasing attention has been paid to VFA and BMI as obesity indexes. Previous studies found VFA to be superior to BMI in accurately and effectively predicting the effect of obesity on adverse postoperative outcomes ${ }^{[22-24]}$ after radical colectomy as well as radical gastrectomy. However, all the studies focused on the comparison of two indications and whether VFA is a better parameter than BMI remains controversial. Similarly, our previous study also only demonstrated that VFA was superior to BMI in predicting PM based on the direct comparison of two variables, thus, we designed this prospective cohort study with the aim of investigating the role of VFA for PM after eliminating BMI differences. To the best of our knowledge, this is the first prospective cohort study focusing on this contentious question.

Despite the controversy of which is better, both VFA and BMI are obesity parameters. There was a positive correlation between BMI and VFA in general in the study although patients with a high BMI may not have a high VFA. Considering that patients were subdivided according to the BMI, the cutoff value for VFA in the previous study was inappropriate for the overall patients. We thus performed ROC curve analysis in all the three BMI groups and cutoff values of $67.28 \mathrm{~cm}^{2}, 88.03 \mathrm{~cm}^{2}$ and $175.32 \mathrm{~cm}^{2}$, respectively, were defined for high VFA, which was quite different from our previous work[16]. However, there is still a lack of consensus on the VFA cutoff for Chinese and VFA cutoff varies from study to study ${ }^{[25,26]}$. Using these cutoffs, $15.52 \%$, $40.15 \%, 31.28 \%$ of patients in our cohort met this definition of high VFA, and these high VFA patients were more likely to develop PM than low VFA patients.

We further confirmed that high VFA was an independent risk factor for PM in the normal BMI group. This may be partly because of the state of low grade chronic inflammation of adipose tissue in obesity. By generating reactive oxygen species (ROS), which have mitogenic properties at low concentrations, tumor further progressed[27,28]. On the other hand, insulin resistance in obese patients may promotes secretion of IGF-1 and thus promote mitogenic and proangiogenic pathways and inhibit apoptosis, consequently cause a majority of the proliferative effects and promote tumor development and metastasis[29]. However, no such results were found in the low and high BMI groups. For patients with low BMI, only $15.52 \%$ of the patients were determined as high VFA. Combined with the low incidence of PM $(6.02 \%, 7$ in 116$)$ in this group, even no factor was found to be independently associated with PM in the multivariate analysis, which may be attributed to statistical bias to some extent. For high VFA patients, the incidence of the PM in the study was the highest $\left(31.28 \%, 61\right.$ in 195), which was also apparently higher than that reported in other studies ${ }^{[30,31]}$. However, the prediction of VFA for PM may be severely weakened by the high BMI of the population. 
Many studies have focused on evaluating the PM status in gastric cancers ${ }^{[31-33]}$. Despite the poor overall sensitivity ${ }^{[34]}$, CT is still the most common tool for detecting PM before operation. Although a previous study ${ }^{[16]}$ already demonstrated the relationship between VFA and PM, this study also found VFA was relevant only in normal BMI patients. As an abdominal CT scan is routinely performed as a common test for preoperative evaluation, the development of software technology also makes VFA measurements easier. Thus, more attentions should be paid to high VFA patients, especially for high VFA and normal BMI patients.

This study has some limitations. First, our study sample size was small, 87 patients were diagnosed with PM and only 7 PM patients were found in the low BMI group, which may seriously affect the accuracy of the analysis. Second, this was a single-center study, and most of the patients came from Southeast China, thus, the findings may not be generalizable to other settings. As body shape is inherently different between locations and races, our results need to be validated by further studies from other medical centers. Third, although ROC curves are appropriate for establishing VFA cutoff values in each group, a standardized cutoff value is urgently needed. Finally, a randomized clinical trial was urgently need to verify the results of this study that reduce VFA may decrease the risk of peritoneal metastasis in GC patients with normal BMI.

In conclusion, our previous study demonstrated VFA is a better obesity index than BMI in predicting abdominal metastasis. This study further determined the relationship between VFA and PM of GC across patients with different BMI. We found that GC patients with high VFA in and normal BMI were at higher risk of PM compared to those with low or high BMI. Thus, more attention should also be paid to GC patients with high VFA and normal BMI. Additionally, for GC patients with normal BMI, intervention measures should be taken to reduce visceral fat area, so as to reduce the risk of peritoneal metastasis.

\section{Declarations}

\section{Ethical statement}

This project was approved by the Ethical Review Board of the Second Affiliated Hospital of Wenzhou Medical University.

\section{Conflict of interest}

None.

\section{Funding}

This study was funded by the National Natural Science Foundation of China (grant no.31670922 and 81672707), and the Wenzhou Basic Scientific Research Projects (grant no. Y20180064).

\section{Conflict of interest:}

All of the authors declare no conflict of interests.

\section{Acknowledgement:}

We thank Editage Group (https://www.editage.cn/) for polishing the draft of this manuscript.

\section{Authorship contribution statement}

Conception or design of the work: XS and CCM; drafting the work: CCM and MMS; data acquisition: MMS; data analysis: HC, LBX; data interpretation: XS and CCM; supervision or mentorship: XS and HH. All the authors contributed important intellectual content for the overall work. XS and CCM take responsibility for the honesty and accuracy of the present study.

\section{Availability of data and materials}

The datasets used and analysed during the current study are available from the corresponding author on reasonable request.

\section{Consent for publication}

The Board of the Second Affiliated Hospital of Wenzhou Medical University approved the publication of this manuscript.

\section{References}

1. Torre LA, Bray F, Siegel RL, Ferlay J, Lortet-Tieulent J, et al. Global cancer statistics, 2012. CA Cancer J Clin. 2015;65: 87-108.

2. Ferlay J, Soerjomataram I, Dikshit R, Eser S, Mathers C, et al. Cancer incidence and mortality worldwide: sources, methods and major patterns in GLOBOCAN 2012. Int J Cancer. 2015;136: E359-386.

3. Qin R, Yang Y, Qin W, Han J, Chen H, et al. The Value of Serum Immunoglobulin G Glycome in the Preoperative Discrimination of Peritoneal Metastasis from Advanced Gastric Cancer. J Cancer. 2019;10: 2811-2821. 
4. Wang C, Shi M, Ji J, Cai Q, Jiang J, et al. A self-enforcing HOXA11/Stat3 feedback loop promotes stemness properties and peritoneal metastasis in gastric cancer cells. Theranostics. 2019;9: 7628-7647.

5. Maehara Y, Hasuda S, Koga T, Tokunaga E, Kakeji Y, et al. Postoperative outcome and sites of recurrence in patients following curative resection of gastric cancer. Br J Surg. 2000;87: 353-357.

6. Glockzin G, Piso P Current status and future directions in gastric cancer with peritoneal dissemination. Surg Oncol Clin N Am. 2012;21: 625-633.

7. Rau B, Brandl A, Piso P, Pelz J, Busch P, et al. Peritoneal metastasis in gastric cancer: results from the German database. Gastric Cancer. 2019.

8. Thomassen I, van Gestel YR, van Ramshorst B, Luyer MD, Bosscha K, et al. Peritoneal carcinomatosis of gastric origin: a population-based study on incidence, survival and risk factors. Int J Cancer. 2014;134: 622-628.

9. Lega IC, Lipscombe LL Review: Diabetes, Obesity and Cancer - Pathophysiology and Clinical Implications. Endocr Rev. 2019.

10. Keum N, Greenwood DC, Lee DH, Kim R, Aune D, et al. Adult weight gain and adiposity-related cancers: a dose-response meta-analysis of prospective observational studies. J Natl Cancer Inst. 2015;107.

11. Arnold M, Pandeya N, Byrnes G, Renehan PAG, Stevens GA, et al. Global burden of cancer attributable to high body-mass index in 2012: a population-based study. Lancet Oncol. 2015;16: 36-46.

12. Lopez-Suarez A Burden of cancer attributable to obesity, type 2 diabetes and associated risk factors. Metabolism. 2019;92: 136-146.

13. Avgerinos KI, Spyrou N, Mantzoros CS, Dalamaga M Obesity and cancer risk: Emerging biological mechanisms and perspectives. Metabolism. 2019;92: 121-135.

14. Aquina CT, Rickles AS, Probst CP, Kelly KN, Deeb AP, et al. Visceral obesity, not elevated BMI, is strongly associated with incisional hernia after colorectal surgery. Dis Colon Rectum. 2015;58: 220-227.

15. Chen XD, Mao CC, Zhang WT, Lin J, Wu RS, et al. A quantified risk-scoring system and rating model for postsurgical gastroparesis syndrome in gastric cancer patients. J Surg Oncol. 2017;116: 533-544.

16. Chen $X$, Chen W, Huang Y, Xu J, Zeng Y, et al. A quantified risk-scoring system including the visceral fat area for peritoneal metastasis of gastric cancer. Cancer Manag Res. 2019;11: 2903-2913.

17. Davis GJ Guidelines for healthy weight. N Engl J Med. 1999;341: 2097-2098.

18. Collaboration NCDRF Trends in adult body-mass index in 200 countries from 1975 to 2014: a pooled analysis of 1698 population-based measurement studies with 19.2 million participants. Lancet. 2016;387: 1377-1396.

19. Obesity: preventing and managing the global epidemic. Report of a WHO consultation. World Health Organ Tech Rep Ser. 2000;894: i-xii, 1-253.

20. Zhou BF, Cooperative Meta-Analysis Group of the Working Group on Obesity in C Predictive values of body mass index and waist circumference for risk factors of certain related diseases in Chinese adults--study on optimal cut-off points of body mass index and waist circumference in Chinese adults. Biomed Environ Sci. 2002;15: 83-96.

21. Chen CM Overview of obesity in Mainland China. Obes Rev. 2008;9 Suppl 1: 14-21.

22. Yang SJ, Li HR, Zhang WH, Liu K, Zhang DY, et al. Visceral Fat Area (VFA) Superior to BMI for Predicting Postoperative Complications After Radical Gastrectomy: a Prospective Cohort Study. J Gastrointest Surg. 2019.

23. Zhai TS, Kang Y, Ren WH, Liu Q, Liu C, et al. Elevated visceral fat area is associated with adverse postoperative outcome of radical colectomy for colon adenocarcinoma patients. ANZ J Surg. 2019;89: E368-E372.

24. Liu Y, Guo D, Niu Z, Wang Y, Fu G, et al. Prediction of the Risk of Laparoscopy-Assisted Gastrectomy by Comparing Visceral Fat Area and Body Mass Index. Gastroenterol Res Pract. 2018;2018: 1359626.

25. Examination Committee of Criteria for 'Obesity Disease' in J, Japan Society for the Study of O New criteria for 'obesity disease' in Japan. Circ J. 2002;66: 987-992.

26. Zhou CJ, Cheng YF, Xie LZ, Hu WL, Chen B, et al. Metabolic Syndrome, as Defined Based on Parameters Including Visceral Fat Area, Predicts Complications After Surgery for Rectal Cancer. Obes Surg. 2019.

27. Ziech D, Franco R, Pappa A, Panayiotidis MI Reactive oxygen species (ROS)--induced genetic and epigenetic alterations in human carcinogenesis. Mutat Res. 2011;711: 167-173.

28. Kim YJ, Kim EH, Hahm KB Oxidative stress in inflammation-based gastrointestinal tract diseases: challenges and opportunities. J Gastroenterol Hepatol. 2012;27: 1004-1010.

29. Samani AA, Yakar S, LeRoith D, Brodt $P$ The role of the IGF system in cancer growth and metastasis: overview and recent insights. Endocr Rev. 2007;28: 20-47.

30. Kim M, Jeong WK, Lim S, Sohn TS, Bae JM, et al. Gastric cancer: development and validation of a CT-based model to predict peritoneal metastasis. Acta Radiol. 2019: 284185119882662.

31. Liu S, He J, Liu S, Ji C, Guan W, et al. Radiomics analysis using contrast-enhanced CT for preoperative prediction of occult peritoneal metastasis in advanced gastric cancer. Eur Radiol. 2019.

32. Chang DK, Kim JW, Kim BK, Lee KL, Song CS, et al. Clinical significance of CT-defined minimal ascites in patients with gastric cancer. World J Gastroenterol. 2005;11: 6587-6592. 
33. Yan C, Zhu ZG, Yan M, Chen KM, Chen J, et al. [Value of multidetector-row CT in the preoperative prediction of peritoneal metastasis from gastric cancer: a single-center and large-scale study]. Zhonghua Wei Chang Wai Ke Za Zhi. 2010;13: 106-110.

34. Wang Z, Chen JQ Imaging in assessing hepatic and peritoneal metastases of gastric cancer: a systematic review. BMC Gastroenterol. 2011;11: 19.

\section{Figures}

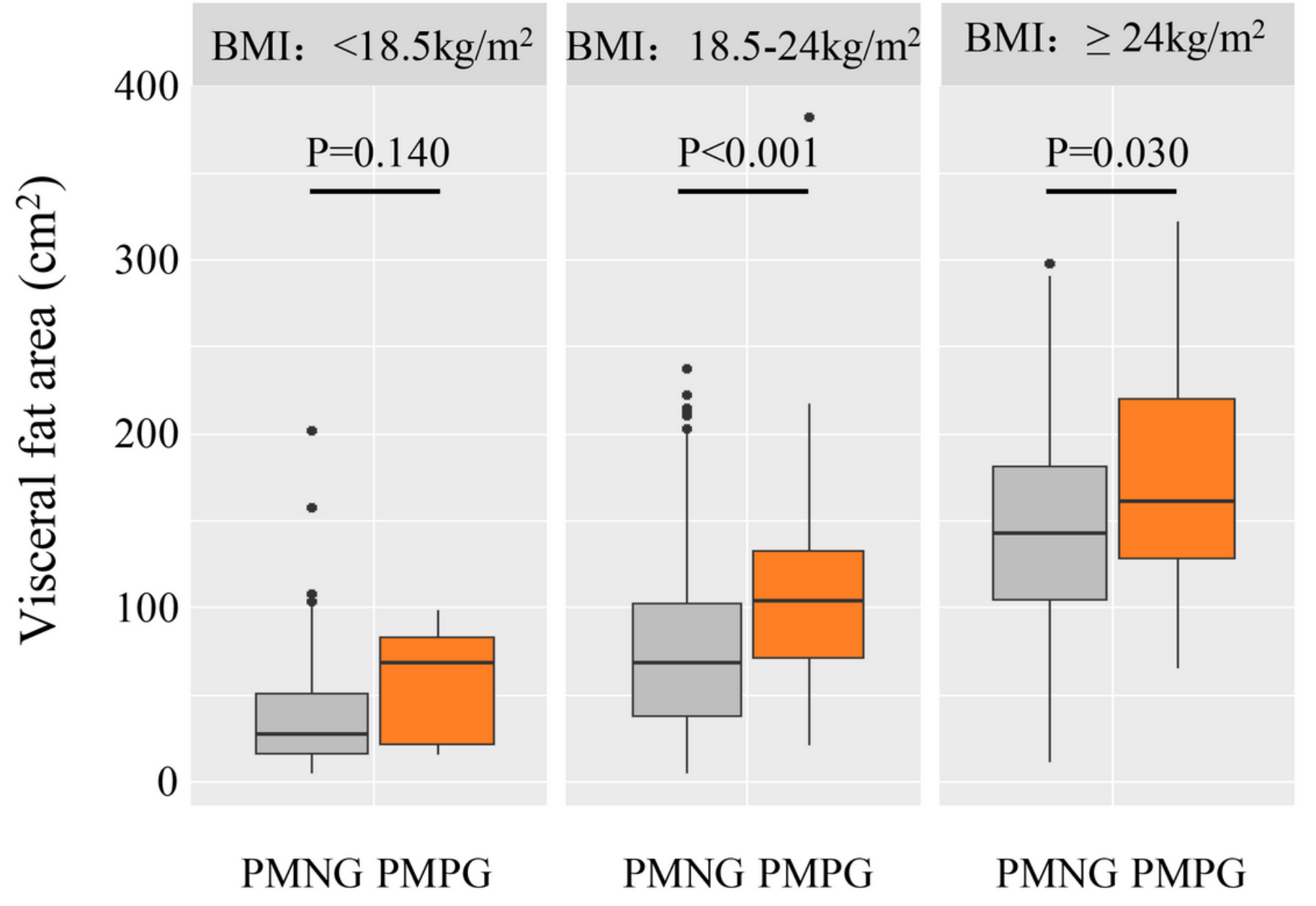

Figure 1

Distribution of VFA between PM and Non-PM in different BMI groups. 
A

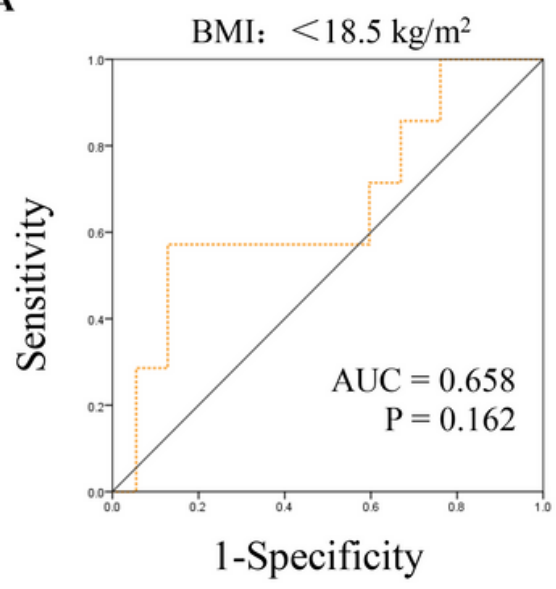

B

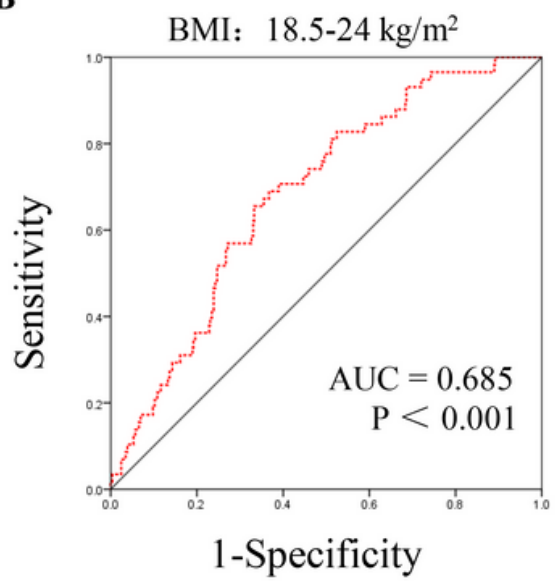

C

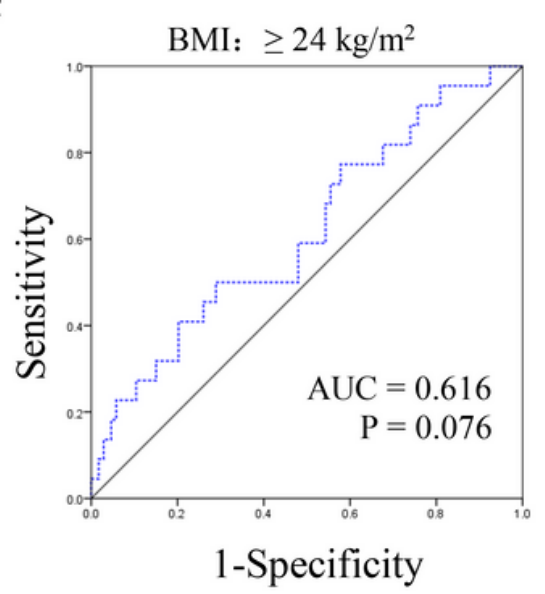

Figure 2

ROC curves to identify PM in different BMI groups. The area under the ROC curve (AUC) values for VFA were shown. 